\title{
Utilidad del drenaje quirúrgico en la cirugía de Artroplastia Total de Rodilla.
}

\author{
DOI: http://dx.doi.org//10.37315/SOTOCAV20202815522 \\ *TECLES-PEYDRO J, **TECLES-TOMÁS JA, * MIFSUT- MIEDES D \\ *UNIVERSIDAD DE VALENCIA DEPARTAMENTO DE CIRUGÍA \\ **HOSPITAL DE XÀTIVA “LLUÍS ALCANYÍS
}

\begin{abstract}
Resumen.
Introducción. El objetivo de este trabajo es estudiar el efecto de la utilización de drenajes quirúrgicos en la cirugía de Artroplastia Total de Rodilla, en cuanto a la aparición de complicaciones asociadas, la necesidad de reintervención, pérdida de sangre y necesidad de transfusión, así como, movilidad postoperatoria.Material y métodos. Se trata de un estudio retrospectivo, observacional, descriptivo en el que 112 pacientes operados de Artroplastia Total de Rodilla se distribuyen en 2 grupos en base a la utilización o no de drenaje. Además de las variables demográficas, se estudiaron las siguientes: Días de estancia postquirúrgica, pérdida de Hemoglobina y de Hematocrito, necesidad de transfusión, complicaciones, y reintervenciones. Resultados. Las características demográficas fueron similares en los dos grupos de estudio. En las variables pérdida de hemoglobina y pérdida de hematocrito se destacaron diferencias estadísticamente significativas $(p<0,05)$ con un mayor descenso en el grupo de pacientes que utilizaron drenaje. Las variables demográficas grado radiológico de Ahlbäck y tipo de prótesis mostraron tener potencial de ser factores modificadores para la pérdida sanguínea, objetivándose un mayor descenso en modelos PS y en grados altos de osteoartritis. En el resto de las variables no se hallaron diferencias relevantes entre los dos grupos.Conclusiones. Este estudio pone de manifiesto la ausencia de beneficio en la utilización de drenajes quirúrgicos en la cirugía de Artroplastia total de rodilla. La utilización de dicha técnica quirúrgica no conlleva ninguna mejoría postoperatoria en comparación con pacientes que no la utilizaron en cuanto a necesidades de reintervención, aparición de complicaciones o limitaciones en la movilidad. De hecho, los drenajes producen una mayor pérdida sanguínea caracterizada por un mayor descenso en la hemoglobina y hematocrito tras la cirugía, pese a que dicha pérdida no conlleva un aumento en la necesidad de transfusión sanguínea.
\end{abstract}

PALABRAS CLAVE: Artroplastia Total de Rodilla, Drenaje Quirúrgico, Osteoartritis, Pérdida Sanguínea.

\section{Summary.}

Introduction. The aim of this work is to study the effect of the use of surgical drains in Total Knee Arthroplasty surgery, regarding the appearance of associated complications, the need for reoperation, blood loss and the need for transfusion, as well as postoperative mobility. Material and methods. This is a retrospective, observational, descriptive study in which 112 patients operated on for Total Knee Arthroplasty are divided into 2 groups based on the use or not of drainage. In addition to demographic variables, the following were studied: Days of post-surgery stay, loss of Hemoglobin and Hematocrit, need for transfusion, complications, and reoperations. Results. Demographic characteristics were similar in the two study groups. In the variables hemoglobin loss and hematocrit loss, statistically significant differences $(p<0.05)$ were highlighted, with a greater decrease in the group of patients who used drainage. The demographic variables, radiological Ahlbäck classification and type of prosthesis, showed potential to be modifying factors for blood loss, showing a greater decrease in PS models and in high degrees of osteoarthritis. In the other variables, no relevant differences were found between two groups. Conclusions. This study shows the absence of benefit in the use of surgical drains in total knee arthroplasty surgery. The use of this surgical technique does not entail any postoperative improvement compared to patients who did not use it in terms of reoperation needs, the appearance of complications or limitations in mobility. In fact, the drains produce a greater blood loss characterized by a greater decrease in hemoglobin and hematocrit after surgery, despite the fact that said loss does not lead to an increased need for blood transfusion. 
TECLES-PEYDRÓ J, Y COLS. Utilidad del drenaje quirúrgico en la cirugía de Artroplastia Total de Rodilla.

\section{Correspondencia:}

Dr. Damián Mifsut Miedes

mifsut_dam@gva.es

\section{Introducción}

Los drenajes quirúrgicos han sido ampliamente utilizados en las cirugías, especialmente las traumatológicas. El principal propósito y beneficio esperado en la utilización de drenajes de succión quirúrgicos radica en la teórica menor acumulación de líquidos en el lecho quirúrgico, lo que facilitaría la cicatrización de la herida. Así, los drenajes conseguirían disminuir la inflamación local, la tensión de la piel y por tanto favorecer la perfusión cutánea, lo que de forma global conllevaría a una menor tasa de complicaciones de la herida quirúrgica. Además, tendría la capacidad de reducir el dolor y conseguir un mejor postoperatorio incluyendo un menor tiempo de recuperación.

En la Artroplastia Total de Rodilla (ATR) sigue siendo una práctica habitual dejar un tubo de succión con el objetivo teórico de disminuir las complicaciones locales. Sin embargo, la literatura reciente pone en discusión su idoneidad. Tras un periodo de debate y controversia entre profesionales, las guías clínicas empiezan a señalar que su utilización no es correcta debido a que el conllevan un mayor número de complicaciones que de beneficios. Las guías aconsejan evitar su utilización, pero en la práctica clínica siguen estando presentes en multitud de cirugías, por lo que estudiar su efecto es importante a la hora de tomar decisiones consensuadas sobre los drenajes quirúrgicos. Tanto la Guía Clínica de la $\mathrm{AAOS}^{1}$ como el $^{2}$ Consenso sobre Infecciones de la SECOT ${ }^{2}$ recomiendan, tras el análisis de estudios clínicos con fuerte evidencia, la no utilización de drenajes de succión quirúrgicos. Como hipótesis de nuestro estudio dijimos que el uso de drenajes quirúrgicos no aporta ningún tipo de ventaja y en consecuencia no es recomendable apoyar su utilización en la cirugía de Artroplastia Total de Rodilla, por ello, nuestro objetivo fue estudiar el efecto de la utilización de drenajes quirúrgicos en la cirugía de Artroplastia Total de Rodilla en base a la aparición de complicaciones asociadas, necesidad de reintervención, pérdida de sangre y necesidad de transfusión, y movilidad postoperatoria.

\section{Material y Métodos}

\section{DISEÑO DEL ESTUDIO}

Se trata de un estudio retrospectivo descriptivo observacional de una serie de 112 casos intervenidos de Artroplastia total de rodilla en nuestro Centro entre enero de 2018 y junio de 2018.

Se establecieron 2 grupos:

1. 59 pacientes intervenidos de Artroplastia total de rodilla primaria con drenaje tipo Redón que se retiró de forma sistemática a las 24 horas de la cirugía.

2. 53 pacientes intervenidos de Artroplastia total de rodilla primaria sin ningún tipo de drenaje quirúrgico.
Todos los casos intervenidos fueron por osteoartritis primaria de la rodilla, quedando excluidos casos secundarios a patologías como Artritis Reumatoide $u$ otras Artropatías. Durante la cirugía se utilizó isquemia del miembro intervenido mediante manguito neumático. Todos los pacientes fueron tratados de forma profiláctica con Heparina de Bajo Peso Molecular. Todos los pacientes recibieron profilaxis antibiótica con 2 gramos de Cefazolina administrados 1 hora antes de la isquemia del miembro a intervenir, a las 8 y 16 horas del postoperatorio.

\section{CARACTERÍSTICAS DE LA MUESTRA}

Con el fin de caracterizar la muestra estudiada y de valorar posibles influencias externas se recogieron las siguientes variables demográficas:

1. Edad.

2. Sexo.

3. Grado de osteoartritis radiológico medido mediante la clasificación de Ahlbäck.

4. Modelo de prótesis utilizada.

5. Uso de Ácido Tranexámico de forma profiláctica en la cirugía.

Las variables dependientes analizadas en los dos grupos con el fin de responder al objetivo del estudio fueron las siguientes:

1. Días de estancia postquirúrgica.

2. Pérdida de Hemoglobina, medida mediante la diferencia entre la Hgb. previa y posterior a la cirugía.

3. Pérdida de Hematocrito, medida mediante la diferencia entre el Hto. previo y posterior a la cirugía.

4. Necesidad de transfusión.

5. Aparición de complicaciones asociadas a la cirugía.

6. Infección tanto de la herida como del implante.

7. Reintervención de la articulación previamente operada.

8. Movilidad posterior a la cirugía, medida mediante los grados de flexión y extensión de la rodilla.

Las muestras para la analítica sanguínea de las que se recogieron los valores previos de $\mathrm{Hgb}$. y Hto., fueron tomadas 2 meses antes de la intervención. Las muestras posteriores a la cirugía fueron tomadas a las 24 horas de esta y al alta hospitalaria. El seguimiento de los pacientes para valorar la aparición de complicaciones tardías y el rango de movilidad fue de 6 meses de media.

Tras llevar a cabo la definición operacional de las variables, se elaboró la tabla de recogida de datos a través de una hoja de cálculo de Microsoft @ Office Excel. Posteriormente, se completó la base de datos recurriendo a la historia 
TECLES-PEYDRÓ J, Y COLS. Utilidad del drenaje quirúrgico en la cirugía de Artroplastia Total de Rodilla.

clínica electrónica de los pacientes mediante el programa informático Orion Clinic.

Se utilizaron dos modelos de implantes. La elección de cada uno de ellos fue tomada por el cirujano principal. Los modelos utilizados fueron los siguientes:

Prótesis con estabilización posterior o tipo PS.

Prótesis con retención de cruzado o tipo CR.

\section{ANÁLISIS DE LOS DATOS}

Para el análisis de los datos se empleó el software IBM SPSS (2019) Statistics for Windows, Version 26.0. Armonk, NY: IBM Corp.

Una vez introducidos los datos se realizó una depuración de los mismos con el objetivo de evitar errores en la transcripción de los valores. Este proceso fue realizado a través de tablas de frecuencias.

El siguiente paso fue caracterizar a la población de forma descriptiva mediante el análisis de las medias de las variables continuas y mediante la prevalencia de las variables cualitativas.

Mediante el análisis estadístico se trató de responder a dos tipos de cuestiones. En primer lugar, la distribución de los factores demográficos en los dos grupos del estudio. En un segundo tiempo, la distribución de las variables dependientes según la utilización o no de drenaje.

La pertenencia a un grupo u otro del estudio se tradujo en una variable categórica dicotómica.

Para compararla con otras variables categóricas dicotómicas se utilizaron tablas cruzadas y se empleó la prueba de Chi-cuadrado de Pearson. En aquellos casos en los que hubiera más de un $15-20 \%$ de casillas con recuento menor que 5, debía utilizarse la prueba exacta unilateral de Fisher.

Para las variables cuantitativas, fue necesario en un primer lugar analizar la distribución de esta, comprobando si seguían una distribución normal o no. Esto se realizó mediante la prueba de Kolmogorov-Smirnov.

Nuestras variables cuantitativas no seguían una distribución normal por lo que para analizarlas fue necesario utilizar test no paramétricos. Para compararlas con una variable categórica dicotómica, al ser muestras independientes se utilizó la Prueba U de Mann-Whitney.

Por último, se analizaron la influencia de los datos demográficos en las variables con diferencias estadísticamente significativas entre los dos grupos de estudio. Se siguió la misma metodología comentada previamente. Apareció un nuevo tipo de contraste en el que se comparaba una variable cuantitativa de distribución no normal con una variable categórica no dicotómica. En este caso se aplicó la prueba de Kruskal-Wallis para muestras independientes.

\section{Resultados}

\section{ANÁLISIS DEMOGRÁFICO}

Entre enero y junio de 2018, 112 pacientes fueron intervenidos de artroplastia total de rodilla en nuestro Centro. La edad media de los pacientes en el momento de la intervención fue de 71 años y 3 semanas. Todos ellos fueron intervenidos como proceso terapéutico de osteoartritis primaria tras fracaso del tratamiento inicial. De ellos, el $65.1 \%$ fueron mujeres y el $34.9 \%$ hombres.

En 59 pacientes se utilizó drenaje quirúrgico tras la intervención, mientras que en 53 de ellos se optó por su no colocación. La decisión de la utilización o no del drenaje correspondía al cirujano principal de cada intervención. 2 tipos de prótesis fueron utilizados. En el $80.3 \%$ de los casos fue utilizada la variante PS mientras que en los casos restantes se utilizó el modelo $\mathrm{CR}$.

Durante la intervención, en 54 pacientes se utilizó ácido tranexámico como medida profiláctica para la hemorragia mientras que en 58 casos no se utilizó. Los criterios de uso o no de antifibrinolíticos no fueron registrados en las historias clínicas.Previamente a la cirugía, los pacientes presentaban diferentes estadios radiológicos de la osteoartritis. Fueron clasificados según la clasificación de Ahlbäck. El $13.4 \%$ presentaba un grado 2, el $56.3 \%$ un grado 3 y el $30.4 \%$ un grado 4 . Esta discrepancia de grados se debe a que el estadio radiológico no fue el criterio de inclusión principal para la cirugía, si no que la decisión terapéutica se basaba en la clínica de los pacientes.

Al analizar los factores demográficos de los pacientes incluidos en el estudio se comprobó que no existían diferencias estadísticamente significativas entre ambos grupos (Tablas I y II).

\section{Resumen de contrastes de hipótesis}

\begin{tabular}{|c|c|c|c|c|}
\hline & Hipótesis nula & Prueba & Sig. & Decisión \\
\hline 1 & $\begin{array}{l}\text { La distribución de } \\
\text { EDAD es la } \\
\text { misma entre } \\
\text { categorias de } \\
\text { Utilización de } \\
\text { drenaje. }\end{array}$ & $\begin{array}{l}\text { PruebaU de } \\
\text { Mann-Whitney } \\
\text { para muestras } \\
\text { independientes }\end{array}$ & ,914 & $\begin{array}{l}\text { Conserve la } \\
\text { hipótesis nula. }\end{array}$ \\
\hline 2 & $\begin{array}{l}\text { La distribución de } \\
\text { Clasificación } \\
\text { Ahlbäckes la } \\
\text { misma entre } \\
\text { categorias de } \\
\text { Utilización de } \\
\text { drenaje. }\end{array}$ & $\begin{array}{l}\text { Prueba U de } \\
\text { Mann-Whithey } \\
\text { para muestras } \\
\text { independientes }\end{array}$ & ,483 & $\begin{array}{l}\text { Conserve la } \\
\text { hipótesis nula. }\end{array}$ \\
\hline
\end{tabular}

Se muestran significaciones asintóticas. El nivel de significación es de 050 .

Tabla I: Test no paramétrico, prueba U de Mann-Whitney para muestras independientes relacionando categorías Edad y Clasificación de Ahlbäck con Uso de Drenaje. 
TECLES-PEYDRÓ J, Y COLS. Utilidad del drenaje quirúrgico en la cirugía de Artroplastia Total de Rodilla.

\begin{tabular}{|c|c|c|c|}
\hline \multicolumn{4}{|c|}{ Pruebas de chi-cuadrado } \\
\hline & Valor & df & $\begin{array}{l}\text { Significación } \\
\text { asintótica } \\
\text { (bilateral) }\end{array}$ \\
\hline Chi-cuadrado de Pearson & $.377^{3}$ & 1 & .539 \\
\hline Chi-cuadrado de Pearson & $2,923^{3}$ & 1 & .087 \\
\hline Chi-cuadrado de Pearson & $2,836^{a}$ & 1 & .092 \\
\hline Chi-cuadrado de Pearson & $1,374^{a}$ & 3 & .712 \\
\hline
\end{tabular}

Tabla II: Estadísticos descriptivos: Pruebas de chi-cuadrado relacionando las categorías Sexo, Modelo de Prótesis, Uso de Ácido Tranexámico y Rangos de Edad respectivamente con Uso de Drenaje.

ANÁLISIS ESTADÍSTICO

En la Tabla III podemos observar las diferentes medias de las variables estudiadas en cada grupo, con y sin drenaje.

\begin{tabular}{|l|l|l|l|}
\hline \multirow{2}{*}{ Necesidad de transfusión } & Si & 4 & 2 \\
\cline { 2 - 4 } & No & 55 & 51 \\
\hline Complicaciones & Si & 9 & 8 \\
\cline { 2 - 4 } & No & 50 & 45 \\
\hline \multirow{2}{*}{$\begin{array}{l}\text { Infección superficial de la } \\
\text { herida }\end{array}$} & Si & 3 & 2 \\
\cline { 2 - 4 } & No & 56 & 51 \\
\hline \multirow{2}{*}{ Reintervención } & Si & 4 & 3 \\
\cline { 2 - 4 } & No & 55 & 50 \\
\hline
\end{tabular}

\begin{tabular}{|l|l|l|}
\hline Uso de drenaje & Si & No \\
\hline & & Media \\
\hline Días de estancia postoperatoria & 3,51 & 3,67 \\
\hline $\begin{array}{l}\text { Perdida de hemoglobina } \\
\text { postquirúrgica (gr/dL) }\end{array}$ & 2,77 & 1,87 \\
\hline $\begin{array}{l}\text { Perdida de hematocrito } \\
\text { postquirúrgica }\end{array}$ & 8,89 & 6,01 \\
\hline Capacidad de flexión en grados & 108 & 106 \\
\hline Capacidad de extensión en grados & -2 & -1 \\
\hline
\end{tabular}

Tabla III: Estadísticos descriptivos: análisis descriptivos de las variables estudiadas según utilización o no de drenaje. 
TECLES-PEYDRÓ J, Y COLS. Utilidad del drenaje quirúrgico en la cirugía de Artroplastia Total de Rodilla.

\section{COMPLICACIONES POSTQUIRÚRGICAS}

En los pacientes intervenidos, se registraron 17 casos con distintas complicaciones ocurridas tanto en el postoperatorio inmediato como de forma más tardía, la mayoría de ellas menores. Destacamos cuatro casos de trombosis venosas profundas, ninguno de ellos con antecedentes de riesgo ni ninguna otra condición conocida que predispusiese a fenómenos trombóticos, todos con un riesgo quirúrgico ASA 2. Un paciente sufrió un proceso de anemia grave 4 meses después de la cirugía, proceso que fue atribuido a la intervención. Tanto en el grupo de drenaje como en los casos sin dicha técnica, las complicaciones aparecieron en el $15 \%$ de los pacientes. No existieron diferencias estadísticamente significativas $(p=0,981)$.

\section{NECESIDAD DE REINTERVENCIÓN}

Del total de 112 pacientes, siete tuvieron que ser reintervenidos de su prótesis total de rodilla, en cuatro de ellos se requirió el recambio por infección del implante y en tres por aflojamiento aséptico de éste. No existieron diferencias estadísticamente significativas respecto al uso de drenaje y la aparición de complicaciones $(p=0,560)$.

\section{APARICIÓN DE INFECCIONES}

Cinco casos fueron registrados por infecciones del implante en la rodilla; dos fueron causados por $S$. aureus sensible a cloxacilina, un caso por $S$. epidermidis, un caso por $P$. aeruginosa y el último por $\mathrm{C}$. Jeikeium. No existieron diferencias estadísticamente significativas entre los grupos drenaje sí y drenaje no, con la aparición de infecciones $(p=0,551)$.

\section{DÍAS DE ESTANCIA POSTOPERATORIO}

La mediana de días de estancia postoperatoria fue de 3,00 con una desviación estándar de 0,954 días. Tanto en los pacientes que utilizaron drenaje como en los que no lo usaron la mediana se mantenía a los 3 días, con una desviación estándar en el primer grupo de 0,878 días y en el segundo de 1,034 días. No existieron diferencias estadísticamente significativas entre los dos grupos (Tabla $\mathrm{V})$.

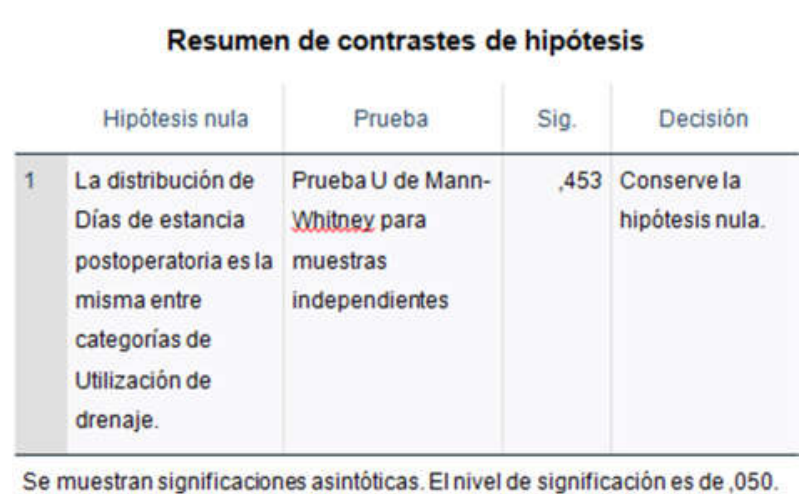

Tabla V: Prueba U de Mann-Whitney para comparar los días de estancia postoperatoria (distribución no normal) con el uso de drenaje.

\section{PÉRDIDA DE HEMOGLOBINA}

La medición de la pérdida de hemoglobina se realizó mediante la resta de los valores de concentración de hemoglobina previa a la cirugía y en los días posteriores. La mediana de la pérdida de hemoglobina en el grupo de pacientes que utilizó drenaje fue de $2,4 \pm 1,24 \mathrm{gr} / \mathrm{dL}$. En el grupo sin drenajes, la mediana del descenso de hemoglobina fue menor, siendo de $1,8 \pm 0,84 \mathrm{gr} / \mathrm{dL}$. La diferencia fue estadísticamente significativa $(p<0,001)$ (Fig. 1).

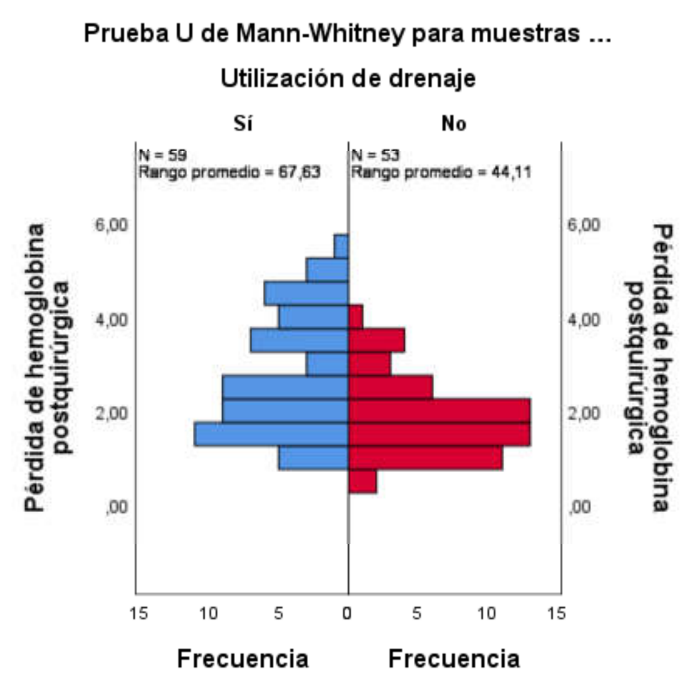

Fig. 1: Prueba $U$ de Mann-Whitney para comparar la pérdida de hemoglobina (distribución no normal) con el uso de drenaje. 


\section{PÉRDIDA DE HEMATOCRITO}

La medición del hematocrito se realizó mediante la resta de los valores de hematocrito previos a la cirugía y los posteriores. La mediana de la pérdida de hematocrito en el grupo de casos en los que se colocó un drenaje fue de $8,5 \pm 3,81$. En el caso de los pacientes a los que no se colocó drenaje postquirúrgico, la mediana de la pérdida de hematocrito fue de $5,8 \pm 2,59$. La diferencia es estadísticamente significativa $(p<0,001)($ Fig. 2$)$.

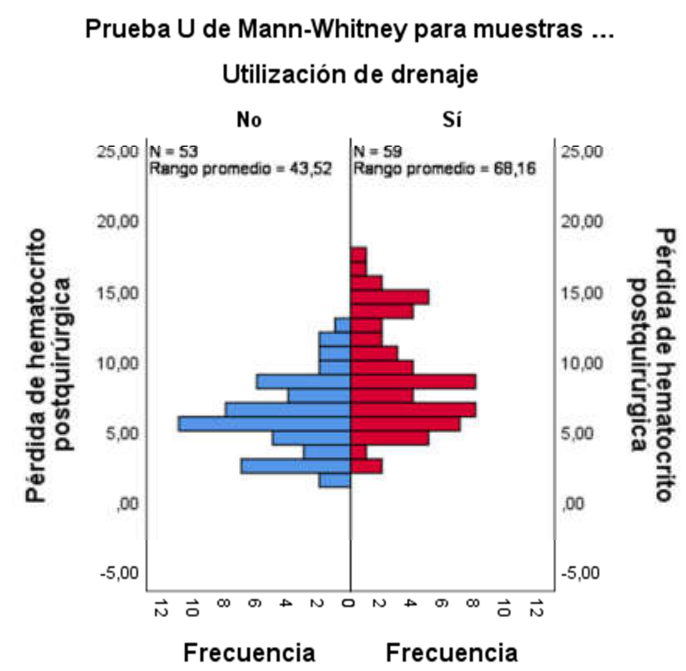

Fig. 2: Prueba $U$ de Mann-Whitney para comparar la pérdida de hematocrito (distribución no normal) con el uso de drenaje.

Dos valores demográficos mostraron relevancia estadística respecto a la pérdida de hemoglobina y/o de hematocrito, uno de ellos fue el modelo de prótesis, encontrándose diferencias estadísticamente significativas con mayor pérdida de hematocrito en aquellos pacientes con modelo de prótesis PS. Este dato podría estar falseado pues el número de casos con modelo PS (90) fue muy superior al de CR (22), por lo que la diferencia podría estar enmascarada por discrepancia. Un estudio clínico prospectivo aleatorizado con doble ciego y similitud de número de casos según modelo sería necesario para comprobar su verdadero efecto en cuanto a la pérdida de hematocrito.

Por otro lado, existieron diferencias estadísticamente significativas tanto para la pérdida de hematocrito como de hemoglobina, si dividíamos los casos según los grados de la clasificación de Ahlbäck. Se objetivó por tanto una pérdida mayor de sangre en aquellas cirugías de articulaciones con mayor grado de osteoartritis radiológica.

No existieron diferencias estadísticamente significativas para la pérdida de hemoglobina y hematocrito en ningún otro apartado demográfico estudiado, con especial interés en la utilización de ácido tranexámico.

\section{NECESIDAD DE TRANSFUSIÓN}

6 pacientes requirieron una transfusión de concentrados de hematíes debido a la pérdida hemática en el postoperatorio inmediato. En todos los casos se transfundieron 2 bolsas de concentrados hemáticos. De todos estos pacientes, 1 presentaba un riesgo aumentado de complicaciones quirúrgicas incluida la necesidad de transfusión. Se trataba de un paciente con mieloma múltiple controlado por hematología y que pese a que se mantenía estable presentaba una anemia preoperatoria con valores de hemoglobina de 10.7 gr/dL. En ningún caso aparecieron complicaciones quirúrgicas que condicionaran un mayor sangrado.

Pese a la mayor pérdida de hemoglobina y hematocrito en el grupo de pacientes con drenaje, no existieron diferencias estadísticamente significativas en cuanto a la necesidad de transfusión en ambos grupos $(p=0,392)($ Tabla VI).

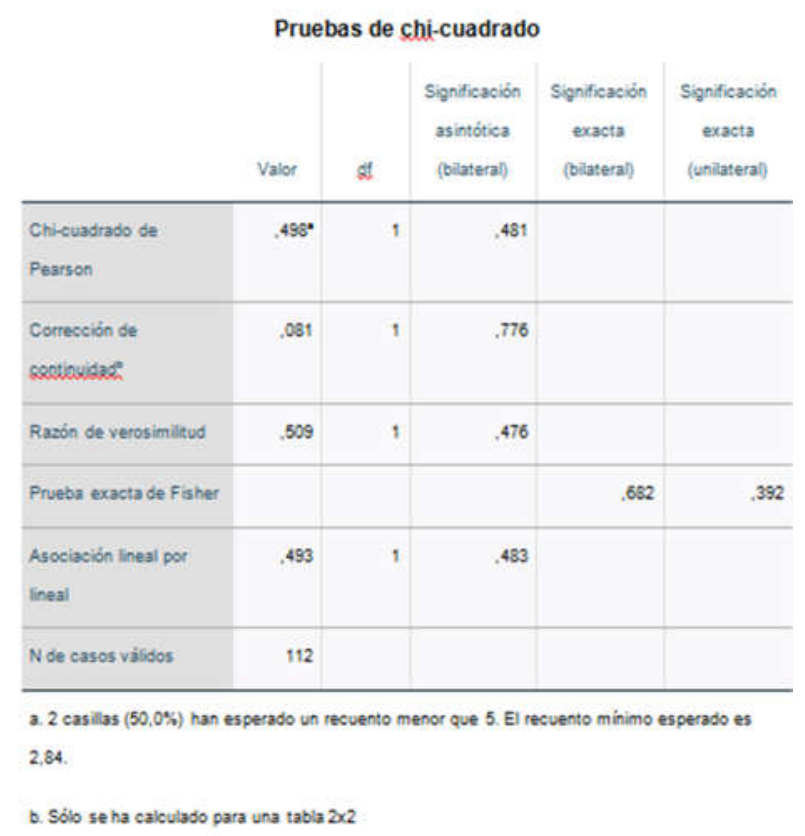

Tabla VI: Estadísticos descriptivos. Prueba de ChiCuadrado comparando la necesidad de transfusión en función del uso de drenaje.

\section{RANGO DE MOVILIDAD}

La flexión de la rodilla intervenida se mantuvo en los pacientes intervenidos en un rango entre 90 y 130 grados. La extensión por su parte alcanzó como máximo los 0 grados y como mínimo 1 paciente solo pudo completar la extensión hasta -30 grados. No existieron diferencias estadísticamente significativas respecto al uso de drenaje y la movilidad postoperatoria tanto en la flexión $(p=0,175)$ como en la extensión $(p=0,432)$. 


\section{Discusión}

Desde que en 1961 Waugh y Stinchfield ${ }^{3}$ propusieran la incorporación de un sistema cerrado de drenaje tras la cirugía de prótesis total de rodilla, este sistema ha sido ampliamente utilizado por los cirujanos. Sin embargo, la reciente literatura no apoya esta metodología. Tanto la Guía Clínica de la $\mathrm{AAOS}^{1}$ como el Consenso sobre Infecciones de la SECOT ${ }^{2}$ recomiendan, tras el análisis de estudios clínicos con fuerte evidencia, la no utilización de drenajes de succión quirúrgicos. Su base teórica principal es la mayor pérdida de sangre que producen estos sistemas al evitar el efecto tapón de la articulación cerrada. De forma añadida, los autores que se posicionan en contra de su utilización argumentan que podrían ser la puerta de entrada de bacterias asociándose con mayor riesgo de infección.

Nuestro estudio sugiere que estas consideraciones son correctas, al no encontrar ningún beneficio estadísticamente significativo en el uso de drenajes quirúrgicos en las artroplastias totales de rodilla.

Jenny, J. Y. et al. ${ }^{4}$ destacan una mayor aparición de equimosis en los pacientes en los que no se usó drenaje, pero ninguno de los casos tuvo gran relevancia clínica por lo que no consideran este proceso como un criterio válido para decidir el uso o no de drenaje. En nuestro estudio, no apareció ningún caso con equimosis clínicamente relevante. En cuanto a complicaciones similares un caso en el que no se utilizó drenaje sufrió un sangrado abundante en los días próximos a la cirugía, y otro paciente que si llevó succión sufrió un hidrartros en la articulación intervenida. En definitiva, ninguna complicación apareció de forma significativamente superior en ninguno de los dos grupos.

Uno de los principios que siguen los cirujanos a la hora de utilizar drenajes es la creencia de que disminuyen la probabilidad de aparición de infecciones profundas. Sin embargo, ningún estudio de la literatura disponible ni tampoco nuestro propio trabajo ha establecido un mayor riesgo de infección profunda al no utilizar drenajes.

De hecho, Li, C. et al. ${ }^{5}$ destacó un mayor número de transfusiones sanguíneas en aquellos pacientes en los que se utilizó la técnica en cuestión. Dichas transfusiones se acompañan de ciertos riesgos como reacciones hemolíticas, anafilácticas 0 incluso desarrollo de infecciones. Por ello, de forma secundaria, y así lo indica también el Consenso sobre Infecciones de la $\mathrm{SECOT}^{2}$, los drenajes suponen un riesgo aumentado de aparición de infecciones. En nuestro caso, sin embargo, no fue necesario, de forma estadísticamente significativa, un mayor uso de transfusiones sanguíneas.

Numerosos estudios ${ }^{4-7}$, de acuerdo con nuestros resultados, destacan una mayor pérdida de sangre al utilizar drenajes quirúrgicos. Esto es comprobable con la mayor pérdida de hemoglobina y pérdida de hematocrito que nuestro estudio ha puesto de manifiesto. La explicación que aporta la literatura sería que tanto la propia articulación como los tejidos blandos vecinos, entre los que destacan los músculos harían una presión suficiente para provocar un efecto tapón que impediría la pérdida de sangre continuada. El drenaje supone una vía de escape para la sangre que no permitiría producir el nombrado tapón de forma efectiva por lo que ocurre una mayor pérdida hemática.

No toda la literatura concuerda con estos hallazgos. De Andrade, M.A.P. et al. $^{8}$ no encontraron diferencias estadísticamente significativas en la pérdida sanguínea al usar o no drenaje. Destacaron que, en el primer mes tras la cirugía, los pacientes que habían llevado drenaje presentaban una mayor movilidad. Esta diferencia no fue tal al ampliar el seguimiento a 6 meses, momento en el que el rango de movilidad era similar en ambos grupos.

Sharma, G.M. et al. ${ }^{9}$, al contrario, destacan una peor movilidad al utilizar drenajes quirúrgicos ya que interfieren con los ejercicios fisioterápicos y secundariamente producen una mayor estancia hospitalaria. Nuestro estudio no destacó diferencias estadísticamente significativas en el rango de movilidad a los 6 meses de la cirugía ni una mayor estancia hospitalaria tras el procedimiento en ninguno de los dos grupos.

Ampliando a otras características técnicas de la cirugía, Jorn, L.P. et al. ${ }^{10}$ y Christodoulou, A.G. et al. ${ }^{11}$ encontraron una mayor pérdida sanguínea al retirar la isquemia del miembro antes del cierre de la herida quirúrgica, comparando con la retirada postoperatoria. Indican que la retirada posterior ofrece mayores ventajas hemostáticas, controlando mejor la actividad fibrinolítica. Además, Sangüesa, $M$. J. et al. ${ }^{12}$ demuestran un menor sangrado al no aplicar vacío a los drenajes durante las primeras 24 horas del postoperatorio. Aplicando esta técnica, la retirada de la isquemia antes o después del cierre de la incisión era indiferente.

En definitiva y como conclusión, las posibilidades técnicas respecto al drenaje son muy diversas y su utilidad y conveniencia se mantiene controvertida. Si bien, parece evidente que el mantenimiento prolongado del drenaje no obtiene ningún beneficio y en cambio produce mayores complicaciones a nivel sanguíneo, el resto de las variables no ofrecen resultados con la suficiente evidencia. Persiste, por tanto, la necesidad de realización de guías clínicas con la suficiente evidencia que marquen el camino a seguir en cuanto a si es necesario y cómo de haberse la utilización de drenajes quirúrgicos.

\section{Conclusiones}

Este estudio pone de manifiesto la ausencia de beneficio en la utilización de drenajes quirúrgicos en la cirugía de Artroplastia total de rodilla. La utilización de dicha técnica quirúrgica no conlleva ninguna mejoría postoperatoria en comparación con pacientes que no la utilizaron en cuanto a necesidades de reintervención, 
TECLES-PEYDRÓ J, Y COLS. Utilidad del drenaje quirúrgico en la cirugía de Artroplastia Total de Rodilla.

aparición de complicaciones o limitaciones en la movilidad. De hecho, los drenajes producen una mayor pérdida sanguínea caracterizada por un mayor descenso en la hemoglobina y hematocrito tras la cirugía, pese a que dicha pérdida no conlleva un aumento en la necesidad de transfusión sanguínea.

\section{Bibliografía}

1. McGrory BJ, Weber KL, Jevsevar DS, Sevarino K. Surgical Management of Osteoarthritis of the Knee: Evidence-based Guideline. JAAOS - J Am Acad Orthop Surg. agosto de 2016; 24(8):e87.

2. SECOT [Internet]. [citado 15 de marzo de 2020]. Disponible en: https://www.secot.es/consenso-internacional

3. Waugh TR, Stinchfield FE. Suction Drainage of Orthopaedic Wounds. JBJS. octubre de 1961; 43(7):939-1021.

4. Jenny J-Y, Boeri C, Lafare S. No drainage does not increase complication risk after total knee prosthesis implantation: a prospective, comparative, randomized study. Knee Surg Sports Traumatol Arthrosc. 1 de septiembre de 2001; 9(5):299301.

5. Li C, Nijat A, Askar M. No Clear Advantage to Use of Wound Drains After Unilateral Total Knee Arthroplasty: A Prospective Randomized, Controlled Trial. J Arthroplasty. 1 de junio de 2011; 26(4):519-22.

6. Closed wound drainage in total hip or total knee replacement. A prospective, randomized study. - Abstract - Europe PMC [Internet]. [citado 15 de marzo de 2020]. Disponible en: https://europepmc.org/article/med/8288663

7. Esler CNA, Blakeway C, Fiddian NJ. The use of a closed-suction drain in total knee arthroplasty: A PROSPECTIVE, RANDOMISED STUDY. J Bone Joint Surg Br. marzo de 2003; 85-B(2):215-7.

8. De Andrade MAP, de Oliveira Campos TV, Silva BFA, de Assis ME, de Castro Boechat L, Biondi LF, et al. Six month follow-up of patients submitted to total knee arthroplasty with and without placement of suction drainage devices. Rev Bras Ortop Engl Ed. 1 de noviembre de 2010; 45(6):549-53.

9. Sharma GM, Palekar G, Tanna DD. Use of closed suction drain after primary total knee arthroplasty - an overrated practice. SICOT-J [Internet]. [citado 15 de marzo de 2020];2. Disponible en: https://www.ncbi.nlm.nih.gov/pmc/articles/PMC5115035/

10. Jorn LP, Lindstrand A, Toksvig-Larsen S. Tourniquet release for hemostasis increases bleeding: A randomized study of 77 knee replacements. Acta Orthop Scand. 1 de enero de 1999; 70(3):265-7.

11. Christodoulou AG, Ploumis AL, Terzidis IP, Chantzidis $P$, Metsovitis SR, Nikiforos DG. The role of timing of tourniquet release and cementing on perioperative blood loss in total knee replacement. The Knee. 1 de agosto de 2004; 11(4):3137 .

12. Sangüesa Nebot MJ, Cabanes Soriano F, Villanueva García E, Fernández Sabaté E. Estudio comparativo de las pérdidas sanguíneas en cirugía protésica primaria de rodilla con diferentes pautas de drenaje y momentos de retirar la isquemia. Rev Ortop Traumatol. 1 de diciembre de 2006; 50(6):437-40. 